\title{
Editors must be vigilant to guarantee the quality and credibility of published scientific work
}

\author{
Manfred Gerlach ${ }^{1}$
}

Published online: 17 October 2018

C) Springer-Verlag GmbH Austria, part of Springer Nature 2018

Scientific journals are designed to communicate primary scientific research and are committed to maintain high standards in order to keep the integrity of the scientific record. The editor-in-chief and the editorial board of a scientific journal are responsible for the entire content of a scientific journal, and it is their responsibility to evaluate the quality and the validity of submitted manuscripts with due respect to the stated aims and standards of their journals. After editorial and peer review, the editor-in-chief ultimately decides whether manuscripts can be published or not. Usually, the editor sends manuscripts for review to independent experts who are not part of the editorial board. Papers may also be rejected without external review, which is at the discretion of the editor. However, there are unfortunately many scientific journals around that do not undertake editorial or peer reviews and at the same time ask for a publication fee (so-called predatory publishing). The Journal Evaluation Tool may be a helpful tool to determine whether a scientific journal is a credible publication source (Rele et al. 2017).

However, based on my long-standing experience as editor-in-chief of ADHD Attention Deficit and Hyperactivity Disorders, there are two editorial difficulties that affect the quality and credibility of published work in scientific journals:

- scientists trying to publish falsified studies, and

- non-scientists trying to publish allegedly scientific studies in scientific journals.

Unbiased, independent and critical assessment is an intrinsic part of all kinds of scholarly work and forms an essential

Manfred Gerlach

manfred.gerlach@uni-wuerzburg.de

1 Department of Child and Adolescent Psychiatry, Psychosomatics and Psychotherapy, Centre of Mental Health, University Hospital of Würzburg, Würzburg, Germany part of an editorial and peer review. This assessment is especially important in the evaluation of papers dealing with Attention Deficit/Hyperactivity Disorder (ADHD), because in the scientific and clinical community there is a lot of discussion including the diagnosis of ADHD, the biological validity of this indication, and its neurodevelopmental origins. Finally, in the public perception, there are many doubts and myths about ADHD.

Recently, there are an increasing number of submissions to ADHD Attention Deficit and Hyperactivity Disorders, especially from the Middle East and Asia, dealing with randomized controlled clinical studies for the treatment of ADHD. Scientific editors' basic assumption is always that authors are submitting studies that are based on honest scientific observation. However, often when I read such papers, I have big doubts as to whether these studies have really been carried out; for example, when I read that ADHD children aged 3-6 years were included in a study and treated with drugs. If I then ask the authors if they would send me copies of the full trial protocol and the letter from the ethics committee that approved the study, I often do not receive any answer.

There are also submissions from authors to ADHD Attention Deficit and Hyperactivity Disorders who are obviously not scientists. Science is related to research that is commonly carried out by academic and research institutions, including government agencies and the industry. A scientist in the field of biology, chemistry, pharmacy or medicine usually holds a degree (i.e., bachelor, master of science, diploma or medical approbation), thus demonstrating that he or she, after years of study, has gathered extensive knowledge and experience in one or more specialty areas. Often, the conduct of research in academic and research institutions as well as in the industry even requires a doctoral degree. Therefore, when I read manuscripts, I always take a critical look at academic titles, degrees, affiliations and the mailing address of the corresponding author in order to get clues about the authors and their experience as scientists. Substantial 
concerns regarding the quality and validity of a paper arise when authors provide only private e-mail addresses.

Unfortunately, editors seem often unaware of the problem that more and more non-scientists try to publish allegedly scientific studies in scientific journals. In this context, a mistakenly published study is noteworthy that linked the most commonly used herbicide glyphosate to the rise of ADHD (Fluegge and Fluegge 2015). This paper was rejected after peer review and consultation with the editorial board of PLoS ONE (The PLoS ONE staff 2015). When reading the affiliations, it seems obvious that at least one of the authors is not an experienced scientist in this field. It is also striking that one of the authors is affiliated to a private institute of health and environmental research. Nevertheless, the authors declared that there were no competing interests. Public trust in the credibility of published studies depends on the critical view of editors and how to deal with potential conflicts of interest. There is a conflict of interest when authors (or their institutions) have financial or personal relationships that may inappropriately influence his or her actions (Uniform requirements for manuscripts submitted to biomedical journals: Writing and Editing Biomedical Publication 2010).

In conclusion, I think that editors have an obligation to only publish studies of high quality and validity. To avoid scientific fraud and the publication of untrustworthy studies with low quality from non-scientists, I strongly recommend that editors-in-chief perform a rigorous pre-screening of submitted manuscripts. In particular, editors-in-chief should be encouraged to review copies of the trial protocol and to check degrees and affiliations of the submitting authors. If there are any doubts about the conduct or integrity of the study, it is their duty to contact the sponsoring institution (Uniform requirements for manuscripts submitted to biomedical journals: Writing and Editing Biomedical Publication 2010).

Acknowledgements I would like to thank my colleagues Prof. Dr. Oliver Tucha and Prof. Dr Andres Warnke for their careful reading of the manuscript and their helpful comments. Finally, I would like to thank Christian von Grafenstein for the linguistic revision.

\section{Compliance with ethical standards}

Conflict of interest Author does not have to report any conflict of interest.

\section{References}

Fluegge KR, Fluegge KR (2015) Glyphosate use predicts ADHD hospital discharges in the healthcare cost and utilization project net (HCUOnet): a two-way fixed-effects analysis. PLoS ONE 10(8):e0133525. https://doi.org/10.1371/journal.pone.0133525

Rele S, Kennedy M, Blas N (2017) Journal evaluation tool. LMU Librarian Publications \& Presentations. 40. http://digitalcom mons.lmu.edu/librarian_pubs/40. Accessed 18 Sept 2018

The PLoS ONE staff (2015) Retraction: glyphosate use predicts ADHD hospital discharges in the healthcare cost and utilization project net (HCUOnet) - a two-way fixed-effects analysis. PLoS ONE 10(8):e0137489. https://doi.org/10.1371/journal.pone.0137489

Uniform requirements for manuscripts submitted to biomedical journals: Writing and editing biomedical publication (2010) J Pharmacol Pharmacother 1:42-58 